\title{
Drug interactions with cannabinoids
}

\author{
Tony Antoniou PhD, Jack Bodkin BScPhm, Joanne M.-W. Ho MD MSc
}

Cite as: CMAJ 2020 March 2;192:E206. doi: 10.1503/cmaj.191097

\section{1}

\section{Cannabinoid levels can be increased by other medications}

Delta-9-tetrahydrocannabinol (THC) and cannabidiol are pharmacologically active cannabinoids in marijuana that are metabolized by cytochrome P450 (CYP)3A4; THC is also metabolized by CYP2C9, a liver enzyme. ${ }^{1} \mathrm{~A}$ pharmacokinetic study found that the CYP3A4 inhibitor ketoconazole nearly doubled THC and cannabidiol concentrations, ${ }^{2}$ and similar interactions could occur with other CYP3A4 inhibitors, including macrolides and verapamil, augmenting the psychoactive effects of THC and dose-related adverse effects of cannabidiol (e.g., somnolence, transaminase elevation). ${ }^{1,2}$ CYP2C9 inhibitors such as cotrimoxazole, fluoxetine and amiodarone would also be expected to increase THC exposure and psychoactive effects. ${ }^{1}$

2

\section{Cannabinoids can affect levels of other drugs}

Cannabidiol inhibits CYP2C19, increasing levels of the active metabolite of clobazam threefold., ${ }^{1,3}$ Interactions with other drugs metabolized by CYP2C19 (Appendix 1, available at www.cmaj.ca/lookup/suppl/ doi:10.1503/cmaj.191097/-/DC1) should be anticipated. Very high international normalized ratio levels and bleeding have been reported with combined used of warfarin and marijuana. ${ }^{1} \mathrm{~A}$ case reporting a threefold increase in tacrolimus levels following the addition of cannabidiol shows that CYP3A4/5 inhibition can also occur. ${ }^{4}$

\section{Smoking marijuana can increase clearance of some drugs}

Smoked marijuana increases the clearance of theophylline $40 \% .{ }^{1}$ Similar findings would be expected for other drugs metabolized by CYP1A2, such as olanzapine. Increased drug clearance occurs with regular marijuana use ( $>2$ marijuana cigarettes per week); no effect of occasional use has been reported.

\section{Additive effects can occur with other drugs}

Additive effects can occur when marijuana is combined with sympathomimetics (e.g., tachycardia, hypertension), central nervous system depressants such as alcohol and opioids (e.g., drowsiness, ataxia), and anticholinergics (e.g., tachycardia, confusion). ${ }^{5}$

5 There are potential "red flag" interactions

Though further research is needed, marijuana may have serious interactions with drugs including warfarin (increased international normalized ratio and risk of bleeding); clobazam (increased risk of benzodiazepine toxicity); central nervous sytem depressants and sympathomimetics (additive effects); and theophylline, clozapine and olanzapine (reduced efficacy). Patients should be advised about possible increased cannabinoid effects with concomitant CYP3A4 and 2C9 inhibitors (Appendix 1). 1,5 Alternatives that do not interact with marijuana should be selected when clinically feasible.

\section{References}

1. Cox EJ, Maharao N, Patilea-Vrana G, et al. A marijuana-drug interaction primer: precipitants, pharmacology, and pharmacokinetics. Pharmacol Ther 2019;201:25-38.

2. Stott C, White L, Wright S, et al. A Phase I, open-label, randomized, crossover study in three parallel groups to evaluate the effect of Rifampicin, Ketoconazole, and Omeprazole on the pharmacokinetics of THC/CBD oromucosal spray in healthy volunteers. Springerplus 2013;2:236.

3. Geffrey AL, Pollack SF, Bruno PL, et al. Drug-drug interaction between clobazam and cannabidiol in children with refractory epilepsy. Epilepsia 2015;56:1246-51.

4. Leino AD, Emoto C, Fukuda T, et al. Evidence of a clinically significant drug-drug interaction between cannabidiol and tacrolimus. Am J Transplant 2019;19:2944-8.

5. Lucas CJ, Galettis P, Schneider J. The pharmacokinetics and the pharmacodynamics of cannabinoids. Br J Clin Pharmacol 2018;84:2477-82.

\section{Competing interests: None declared.}

This article has been peer reviewed.

Affiliations: Department of Family and Community Medicine (Antoniou), St. Michael's Hospital and University of Toronto; Li Ka Shing Knowledge Institute (Antoniou), St. Michael's Hospital, Toronto, Ont.; Department of Medicine (Bodkin, Ho), McMaster University, Waterloo, Ont.; Grand River Hospital (Bodkin), Kitchener, Ont.; Schlegel Research Institute for Aging (Ho), Waterloo, Ont.

Correspondence to: Tony Antoniou, Tony.Antoniou @unityhealth.to 